\title{
Effectiveness of Cognitive-Behavioral Therapy on Premenstrual Syndrome Through Compliance to Treatment in an Iranian Sample
}

\author{
Setareh Babajani, ${ }^{1,}{ }^{*}$ Karim Asgari, ${ }^{1}$ Hamid Reza Orayzi, ${ }^{1}$ and Nezamaddin Ghasemi ${ }^{2}$ \\ ${ }^{1}$ Department of Psychology, University of Isfahan, Isfahan, IR Iran \\ ${ }^{2}$ Department of Psychology, University of Salman Farsi Kazeron, Kazeron, IR Iran \\ "Corresponding author: Setareh Babajani, Department of Psychology, University of Isfahan, Isfahan, IR Iran. E-mail: s.babajani92@gmail.com
}

Received 2017 January 27; Revised 2017 May 13; Accepted 2017 June 09.

\begin{abstract}
Background: This study aims to investigate the effectiveness of cognitive-behavioral therapy on reducing the symptoms of premenstrual syndrome based on the moderator variable of therapy compliance in an Iranian sample.

Methods: This was a semi-experimental study, in which 56 patients with premenstrual syndrome disorder were selected using the accessible sampling method. They were all the female patients who had been referred to the gynecologic and psychiatric centers in Isfahan city, and were randomly assigned into two experimental and control groups, each one comprising 28 patients. The experimental group received 10 sessions of cognitive-behavioral therapy. The patients were tested before and after intervention using the screening questionnaires of premenstrual syndrome symptoms. Additionally, subjects in both experimental and control groups were divided into two groups based on the rate of their therapy compliance (from high to low or noncompliant). Data was Analyzed using of covariance and Cohen's size effect with SPSS-22.

Results: The results showed that the effectiveness of cognitive-behavioral therapy on reducing the symptoms of premenstrual syndrome was statistically significant. Moreover, research findings have shown that the therapy was more effective on the compliant group.

Conclusion: According to the results, cognitive behavior therapy can be suggested as an effective therapeutic approach in reducing the symptoms of premenstrual syndrome, especially for the patients who are complient.
\end{abstract}

Keywords: Premenstrual Syndrome (PMS), Cognitive-Behavioral Therapy, Therapy Compliance

\section{Background}

Menstruation is one of the most important signs of normal performance of the reproductive system in adult females. However, sometimes, it may be associated with other signs, causing physical and mental problems for women. This kind of physical, mental, and emotional symptoms that appear before menstruation and disappear at the beginning of the menstruation period is called premenstrual syndrome [1].

Premenstrual syndrome is characterized by physical, cognitive, mood, and behavioral changes that periodically occur in the luteal phase of the menstrual cycle [2]. These symptoms disappear either at the beginning of the menstruation or after it, and followed by a period with no symptom [3]. The most common symptoms of the premenstrual syndrome are anxiety, depression, fatigue, nervousness, and irritability, feelings of loss of control, confusion, changes in sleep and appetite patterns, headache, breast swelling and tenderness, joint or muscle pains [4]. This syndrome have adverse effects on the life of women and hence, their daily activities at home, personal relationships, social activities, leisure time, sexual affairs, and their work efficiency will be affected by the syndrome [5].

In the mid-1980s, criteria for a severe form of PMS was proposed and confirmed by the DSM-III entitled late luteal phase dysphoric disorder. In the fourth edition of the DSM, this title was replaced by premenstrual dysphoric disorder and diagnostic criteria were slightly modified. However, this category was considered as an appendix. In DSM-5, and the authors separated this disorder from the mood disorders and proposed it as a new diagnostic category $[6,7]$. The prevalence rate of the symptoms is different with respect to the factors such as culture, attitude, age, exercises, and nutrition and background diseases. The findings of a metaanalysis of epidemiology of the premenstrual syndrome in a wide range of papers in 2014 shown that the overall prevalence of premenstrual syndrome is $47.8 \%$; the minimum and maximum rate were $12 \%$ and $98 \%$ in France and Iran, respectively.

Since the cause of the disease in unknown, the syndrome is actually treated symptomatically. The purpose of PMS treatment is reducing the symptoms and their adverse effects on the women's health and promoting quality of life in patients.

Sadock [8] reported that $3 \%-7 \%$ of women might be 
affected by premenstrual dysphoric disorder. Severity of symptoms thought to be different between the premenstrual dysphoric disorder and late luteal phase dysphoric disorder.

Premenstrual dysphoric disorder (PMDD) can be changed into depression [8]. PMDD is a disease occurring whenever the steroid hormone levels is changed in menstruation-ovulation cycle and is characterized by irritability, emotional instability, headache, anxiety, and physical symptoms [8]. Dysfunction in hypothalamuspituitary- adrenal axis observing in depressive patients is also reported in PMDD patients [9].

Although the etiology of PMS and PMDD is not clear. Some studies proposed a possible role of the automatic nervous system in its pathology and it was reported that women with PMDD have a higher level of no epinephrine compared to that of normal women [1].

The syndrome which is characterized by mood or emotional disturbances is of great importance since quality of life can be changed by mood swing. Hence premenstrual syndrome as a kind of mood disorder can be affecting on cognitive functions in patients $[10,11]$. Blake et al. (1998) studied the use of cognitive-behavioral therapy for the treatment of premenstrual syndrome [3]. They believed that women with premenstrual syndrome might negatively interpret the psychological events. The cognitive-behavioral therapy helps the women find more compatible ways to deal with premenstrual changes [1214]. According to the ACOG (2000), medication is the first treatment method for Premenstrual Dysphoric Disorder. However, non-pharmaceutical strategies should be considered as the first solution for different kinds of mild premenstrual syndrome [15]. Many authors, including Kirkby (1994), Christensen (1995), Blaker et al. (1998) [1], Haynes et al. (1998) [13], Navabinejad and Davoodvandi [16] and Davoodvandi [6] (2011) considered cognitivebehavioral therapy as one the most eligible treatments for premenstrual syndrome. Others, such as Feldman (2007), Dugas (2003), Hoffman (2007), and Marlatt at c (2007) believe that cognitive-behavioral therapy in the treatment of those emotional and physical disorders accompanying with symptoms with premenstrual syndrome (e.g., pain and anxiety) played a key role in proposing a theoretical framework for the treatment of the premenstrual syndrome [14].

Therapy compliance is an important variable, in predicting the outcomes of therapy. Compliance in medicine indicates how the patients follow the prescribed therapeutic program of the therapist or their effective treatment [15]. Non-compliance is the failure or refusal to comply and can be defined as a type of disobedience to the therapist. The process of treatment involves stages like seeking and receiving therapy, and hence, in different stages of therapy, the patients may leave the treatment process [16]. Royal Pharmaceutical Society of Great Britain has replaced compliance with concordance suggesting an agreement and harmony and not merely compliance. Adherence was interchangeably used with compliance as well $[17,18]$. A wide variety of psychological, social, and medicinal factors may affect the patients' therapy compliance [19].

Although therapy compliance as one of the main variables plays a significant role in predicting the treatment outcomes, but it has been ignored, or left without much attention in previous studies. Indeed, those studies have regarded compliance basically in pharmacotherapy and disregarded the role of this important variable in predicting the results of the psychological treatments [9]. The role of the therapy compliance that refers to the individual differences in various treatments has been dealt with in pharmacotherapy. In this study, the authors aimed to investigate the role of compliance in psychological treatments by considering the moderating role of the therapy compliance in the effectiveness of cognitive-behavioral therapy for patients with premenstrual syndrome.

\section{Methods}

This was a semi-experimental study, in which the patients who had diagnosed with PMS, by a physician, were screened by premenstrual symptoms screening questionnaire. They were scored as the medium level of the syndrome, and were divided into control and therapy groups. Written consent was obtained from all of the patients. Moreover, the authors developed a questionnaire for therapy compliance which used in order to divide the control and treatment groups into two groups of high compliance and low or noncompliant using the mean score of the questionnaire. Then the patients in therapy group were assessed by the questionnaires and received the cognitivebehavioral therapy for 10 sessions. Participants were reassessed at the end of the therapy sessions by the same questionnaires as the posttest phase (Tables 1 and 2).

At first, patients with premenstrual syndrome were selected using the available sampling and then were assigned randomly into two groups of control and therapy. First, 35 patient were chosen as a sample which at the end of sessions, ultimately, with loss of sample size 28 people for both of the groups were picked. The sample was divided into two groups through the therapy compliance questionnaire. Two groups are selected based on the scores of the people in therapy compliance scale and the mean score used as the cut point. The sample size of this study includes 56 people which their mean age was 24 and standard deviation 12.27 of them were single and 29 of them were mar- 
Table 1. Research Design Diagram

\begin{tabular}{|c|c|c|c|c|c|}
\hline Group & Number & Random Selection & Pretest & Intervention & Posttest \\
\hline Treatment & 28 & $\mathrm{R}$ & $\mathrm{T} 1$ & $\mathrm{X}$ & $\mathrm{T} 2$ \\
\hline Control & 28 & $\mathrm{R}$ & $\mathrm{T} 1$ & - & $\mathrm{T} 2$ \\
\hline
\end{tabular}

Table 2. Subjects in Two Groups of with and without Therapy Compliance

\begin{tabular}{lcc}
\hline Treatment & $=\mathbf{1 4 n}$ & $=\mathbf{1 4 n}$ \\
\hline Control & $=14 \mathrm{n}$ & $=14 \mathrm{n}$ \\
$\begin{array}{l}\text { Low rate of therapy } \\
\text { compliance }\end{array}$ & $\begin{array}{c}\text { High rate of therapy } \\
\text { compliance }\end{array}$ & \\
\hline
\end{tabular}

ried. level of their education include 7 diploma, 44 bachelor and 5 of them were M.A. inclusion and exclusion criteria included lack of severe mental illnesses such as psychosis, lack of disabilities, and chronic physical illnesses, non- participation in other educational and psychotherapy programs and recognition of premenstrual syndrome disorder by specialist.

\subsection{A summary of Treatment Sessions}

Intervention protocol(CBT) of the paper Hosseinnazari et al. (2012) and books were taken step by step guide for premenstrual syndrome [16].

Session 1: explanations about holding the sessions, regulations, training (normal menstruation cycle, premenstrual syndrome, prevalence and differential diagnosis and psychological treatment), self-monitoring training of the activity

Session 2: three-system model of human beings' emotions with $\mathrm{ABC}$ training, Guided Relaxation Imagery

Session 3: defining the automatic thoughts and their effect on the mood and feelings, the characteristics of the automatic thoughts, acquaintance with cognitive distortions or logical errors and the personal ability for their identification in the one's thoughts

Session 4: training the strategies for identifying the thoughts and the ways to obtain them, acquaintance with the nature of schema and their relationships with automatic thoughts

Session 5: providing a main list of the beliefs and classifying them based on a scale of mental inconvenience and the rate of emotion

Session 6: accepting the changing nature of the beliefs, training the assessment methods and modifying the inefficient thoughts, helping the members in raising the questions in order to challenge the automatic thoughts
Session 7: training the Jacobson's progressive muscle relaxation technique and using this technique for reducing the anxiety

Session 8: training the logical analysis about the beliefs and challenging them, practicing the steps of logical analysis

Session 9: members associate a hierarchy of their emotional situations before menstruation and think of it, then, replace them with pleasant situations. Training the problem-solving skill and its uses in daily life

Session 10: Summarizing the content of the sessions, explaining about the probability of the reappearance of the symptoms after the treatment period and proper reaction when reappearing the symptoms, completing the posttest questionnaires.

\subsection{Research Tools}

Premenstrual symptoms screening questionnaire (PSSQ): the premenstrual symptoms screening tool (PSST) is a simple user friendly screening tool to identify women who suffer from severe PMS (premenstrual syndrome)/PMDD (premenstrual dysphoric disorder) and who are likely to benefit from treatment. The PSST is a 19-item instrument consisting of two domains: the first domain includes 14 items related to psychological,physical, and behavioral symptoms and the second domain (five items) evaluates the impact of symptoms on women's functioning. Each item is rated on a fourpoint scale (not at all $=0$, mild $=1$, moderate $=2$, severe $=3$ ). According to the instruction of the PSST devised by Steiner et al. (2003) for diagnosis of PMS, from the following 14 symptoms [13]. [(1) tension/anxiety, (2) irritability/anger, (3) depressed mood/hopelessness, (4) tearful/increased sensitivity to rejection, (5) decreased interest in work activities, (6) decreased interest in home activities, (7) decreased interest in social activities, (8) difficulty concentrating, (9) fatigue/lack of energy, (10) overeating/food cravings, (11) insomnia, (12) hypersomnia, (13) feeling overwhelmed, and (14) physical symptoms], women must report at least fivesymptoms as moderate or severe where at least one should be from symptoms numbers 1 - 4 (namely core symptoms). Also, they must report if their symptoms interfere moderately or severely with their ability to function in at least one of five items in 
the second domain [(a) work efficiency, (b) relationships with coworker (c) relationships with family, (d) social life activities, and (e) home responsibilities]. For diagnosis of PMDD, the following criteria must be present: (1) at least one of the symptoms ( 1 to 4 ) as severe; (2) in addition, at least four of the symptoms ( 1 to 14) as moderate to severe; and (3) at least one of a, b, c, d, and e as severe [13]. The PSST has been used in different countries. Validated translations of this questionnaire are available in Hindu for India, Polish, Italian, and Spanish for Venezuela, Korean, Chinese (mainland), Swedish, and Thai. No validated translations are available in Chinese for Taiwan, French, German, Japanese, Portuguese, Romanian, and Spanish (Flintbox 2012; Tschudin et al. 2010; Chayachinda et al. 2008; Kayatekin et al. 2008; nTschudin et al. 2007; Takeda et al. 2006) [15]. Siahbazi et al reported good reliability and validity for the questionnaire in Iranian population. The reliability of this questionnaire was reported as 0.9. Content validity and content validity index were 0.7 and 0.8 , respectively, confirming its usefulness for our study [20] Also Bakhshani, Mousavi, Khodabandeh (2008) reported good reliability and validity in Iranian female university students

Therapy compliance questionnaire: in order to determine its reliability and validity, four sample groups of women who had been referred to the gynecological clinics, in Beheshti, and Asgarieh hospitals in Isfahan city, were selected. The sample sizes were 134, 42,102, and 9, respectively. The first form of questionnaire comprised of 48 items, which reduced to 16 , after carrying out factor analysis.

Factor analysis was conducted based on orthogonal factorial rotation and varimax method that has the particular value of 4.22, the clarifying variance of 0.91 . (Radolf, 1977; Oreizi, 1387) [19]. Lache validity coefficient for criteria validity was obtained as 0.99 , through consulting with nine lecturers of the School of Nursing and Midwifery, in Isfahan University of Medical Sciences.

\section{Results}

Cognitive-behavioral therapy was found to be effective on the symptoms of the Premenstrual Dysphoric Disorder. Results default equality of variances (Levin test) for treatment is given in the Tables 3 and 4 .

Table 3. Levin Test

\begin{tabular}{cccc}
\hline Sig & Df2 & Df1 & F \\
\hline $\mathbf{0 . 0 1 4}$ & 54 & 1 & 6.49 \\
\hline
\end{tabular}

Cognitive-behavioral therapy was statistically significant on the rate of the premenstrual syndrome in therapy group compared to the control group $(\mathrm{Sig}<0.01)$. Eta 2 was found as 0.50 and Observed power was one. Both of them were statistically desirable. So cognitive- behavioral therapy was effective on the symptoms of the PMDD (Table 5).

Therapy compliance was statistically significant on the rate of the premenstrual syndrome of the patients in posttest compared to the pretest in the treatment group compared to the control group (Sig < 0.01). Eta2 was 0.21 and Observed power was 0.95. Both of them were statistically desirable (Table 6).

As shown in Table 6, there was a statistically significant difference between both groups of high rate therapy compliance and low rate therapy compliance after treatment with cognitive-behavioral therapy.

\section{Discussion}

The findings showed that the effect of cognitivebehavioral therapy on the rate of premenstrual syndrome of the subjects in posttest comparing with the pretest was statistically significant in the treatment group compared to the control group. This was consistent with the reports of Taghizadeh et.al. (2013) that observed a significant reduction of the symptoms of premenstrual syndrome, anxiety, anger and physical symptoms of the patients following conducting a cognitive-behavioral training intervention [21]. Davoodi et.al. (1391) conducted a study with the aim of investigating the cognitive-behavioral therapy, and their results were similar with this study [7]. Moreover, the results of this study were congruent with those of Nazari et.al. (1391), and Davoodvandi and Navabinejad (1390) [6, 22]. Also the results of this study were consistent with those of the reports of Obrien et al. (2007), Salamat et al. (2007), and Hunter et al. (2002) confirming the effectiveness of the cognitive-behavioral therapy on premenstrual syndrome $[10,17,23]$. An interesting finding of our study suggest a positive effect of compliance on the symptoms of premenstrual syndrome, showing that patients with high rate of compliancy had better outcomes following therapy.

In the medical conditions, compliance to the therapist was regarded as an important factor in patient's therapeutic outcome. A patient with high compliance have usually positive attitude toward medications and other forms of therapy, and willingness to meet with the therapist; while, non-compliant patients may forget therapeutic sessions, or not attending in on-time [24]. Medical non-compliance was recognized as a major health problem, imposing considerable financial burdens on health care system.

The significance of therapy compliance in physical diseases like hypertension and diabetes, were investigated in 
Table 4. Covariance Analysis of Cognitive-Behavioral Therapy on the Symptoms of PMDD

\begin{tabular}{lccccccc}
\hline Source & Type III Sum of Square & df & Mean Square & F & P Value & Partial Eta Squared & Observed Power \\
\hline Pre-test & 1256.30 & 1 & 1256.30 & 55.081 & 0.001 & 0.51 \\
Group Membership & 1223.95 & 1 & 1223.95 & 53.66 & 0.001 & 0.50 & 1 \\
\hline
\end{tabular}

Table 5. Covariance Analysis of Therapy Compliance on the Symptoms of PMDD

\begin{tabular}{lccccc}
\hline Source & Type III Sum of Square & df & F & P Value & Partial Eta Squared \\
\hline Pre-test & 1032.29 & 1 & 28.43 & 0.01 & 0.34 \\
Therapy compliance & 508.51 & 1 & 14.006 & 0.01 & 0.21 \\
\hline
\end{tabular}

Table 6. The Effect Size of Cognitive-Behavioral Therapy on PMDD in Both Groups (High and Low Rate of Therapy Compliance)

\begin{tabular}{l|c|c|c|c|c|c}
\hline Therapy Compliance & df & F & Sig & Observed Power & Partial Eta Squared & Effect size \\
\hline Low rate of therapy compliance & 1 & 8.64 & 0.01 & 0.81 & 0.26 & 1.15 \\
\hline Error & 26 & & & & & 0.67 \\
\hline High rate of therapy compliance & 1 & 26.28 & 0.01 & 1 & & \\
\hline Error & 26 & & & & & \\
\hline
\end{tabular}

recent researches, suggesting its importance in the course of therapy [4]. However, its importance seems to be neglected in many psychiatric diseases, including PMS.

Since the therapy non-compliance may lead to undesirable consequences like exacerbation of the symptoms or termination of the therapy, it is necessary to think about it, and to its importance in medical treatments, since it is associated with irreparable consequences, the effective factors on the rate of therapy compliance is examined in the studies and solutions for increasing the rate of the therapy compliance are sought [2]. In one of the studies, mood characteristics of the patients with diabetes and their therapy compliance were taken into consideration. In that study, mood irritability was one of the main factors. Another study investigated the lack of the guidance for the patients as the most effective factor in the therapy noncompliance. This study showed that the patients thought that they have been treated and recovered, and this was another important factor in their therapy non-compliance [15].

The size effect for all groups is desirable, namely, cognitive-behavioral therapy on the premenstrual syndrome has been effective in both groups of high and low rate of therapy compliance. However, the effectiveness rate has been different so that there is the high effect size in the groups with high rate of therapy compliance and there is lower effect size in the groups with low rate or no therapy compliance.
In practice, this study showed that intervention has been successful in both groups of high and low rate of the therapy compliance. However, in the group with high rate of therapy compliance, the treatment was more effective. Consequently, as therapy compliance has been regarded as one of the main variables in recovery of the patients in medical treatments, it is of great importance in psychological treatments too and they should be considered in psychological treatments as they are taken into consideration in medical treatments.

As the medical studies and this study on the problems and psychological treatments shown, therapy compliance plays a crucial role in the treatment, process and the outcomes in the patients. There are different other factors that affect the therapy compliance, among them are social support by family, economic conditions and social isolation [15].

\section{Acknowledgments}

This research was part of the master's thesis of Isfahan University, Isfahan, Iran.

\section{Footnotes}

Authors' Contribution: All authors had equal role in design, work, statistical analysis and manuscript writing. 
Conflict of Interest: The authors declare that there was no conflict of interests in this study.

\section{Funding/Support: Isfahan University.}

\section{References}

1. Baker FC, Colrain IM, Trinder J. Reduced parasympathetic activity during sleep in the symptomatic phase of severe premenstrual syndrome. J Psychosom Res. 2008;65(1):13-22. doi: 10.1016/j.jpsychores.2008.04.008. [PubMed: 18582607].

2. Balaha MH, Amr MA, Saleh Al Moghannum M, Saab Al Muhaidab N. The phenomenology of premenstrual syndrome in female medical students: a cross sectional study. Pan Afr Med J. 2010;5:4. [PubMed: 21120003].

3. Blake F, Salkovskis P, Gath D, Day A, Garrod A. Cognitive therapy for premenstrual syndrome: a controlled trial. J Psychosom Res. 1998;45(4):307-18. [PubMed: 9794277].

4. Clark N, Jones P, Keller S, Vermeire P. Patient factors and compliance with asthma therapy. Respir Med. 1999;93(12):856-62. [PubMed: 10653046].

5. Connolly M. Premenstrual syndrome: an update on definitions, diagnosis and management. Adv Psychiatr Treat. 2001;7(6):469-77. doi: 10.1192/apt.7.6.469.

6. Davoodvandi M, Navabinejad S, Lotfi Kashani F. Effectiveness of cognitive-behavioral group training on reducing the symptoms of premenstrual syndrome. Med Sci I Islamic Azad Univ. 2011;2(21):114-20.

7. Davooidi A, Izadi Maziri S, Mehrabizadeh Honarmand M. Effectiveness of Group carrative/cognitive-behavioral therapy on premenstrual syndrome of the female students. Iran J Nurs Midwifery Res. 2011;15(11):7-15.

8. Sadock BJ, Sadock VA, Ruiz P. Comprehensive textbook of psychiatry 7th ed. Philadelphia: Williams and wilkins; 2000

9. Parry BL, Javeed S, Laughlin GA, Hauger R, Clopton P. Cortisol circadian rhythms during the menstrual cycle and with sleep deprivation in premenstrual dysphoric disorder and normal control subjects. Biol Psychiatry. 2000;48(9):920-31. [PubMed: 11074230].

10. Hunter MS, Ussher JM, Browne SJ, Cariss M, Jelley R, Katz M. A randomized comparison of psychological (cognitive behavior therapy), medical (fluoxetine) and combined treatment for women with premenstrual dysphoric disorder. J Psychosom Obstet Gynaecol. 2002;23(3):193-9. [PubMed: 12436805].
11. Javaheri R, Neshat-Doost HT, Molavi H, Zare M. Efficacy of cognitivebehavioral stress management therapy on improving the quality of life in females with temporal lobe epilepsy. Arak Med UnivJ. 2010;13(2).

12. Alden KR, Lowdermilk DL, Cashion MC, Perry SE. Maternity and Women's Health Care-E-Book. Elsevier Health Sciences; 2013.

13. Haynes RB, McKibbon KA, Kanani R, Brouwers MC, Oliver T. Inter ventions to assist patients to follow prescriptions for medications (Cochrane review). Cochrane Lib. 1998(3).

14. Lustyk MK, Gerrish WG, Shaver S, Keys SL. Cognitive-behavioral therapy for premenstrual syndrome and premenstrual dysphoric disorder: a systematic review. Arch Womens Ment Health. 2009;12(2):85-96. doi: 10.1007/s00737-009-0052-y. [PubMed: 19247573].

15. Nadeem M, Zaffar U, Ullah MI, Khalid MR, Sheikh SI, Ahmed SI. Factors responsible for non compliance to treatment in pulmonary tuberculosis. J Rawalpindi Med College (JRMC). 2014;18(1):11-2.

16. Navabinejad S, Davoodvandi M. Step by step manual of physical and mental problems of menstruation. Tehran: PErkas Publication; 2013.

17. Indusekhar R, Usman SB, O'Brien S. Psychological aspects of premenstrual syndrome. Best Pract Res Clin Obstetr Gynaecol. 2007;21(2):20720

18. Olive DL, Palter SF. In: Novaks gynecology. Berek JS, editor. Philadelphia: Lippincott Williams \& Wilkins; 2006. pp. 161-84.Reproductive physiology.

19. Oreizi H,Farahani H. Applied methods in clinical psychology and consultancy. Tehran: Vazheh Publication; 2008.

20. Siahbazi S, Hariri F, Montazeri A, Moghadam Banaem L. Standardization of the Premenstrual Symptoms Screening Tool. Translation, and psychometrics of Iranian sample. Payesh J. 2011;10(4):421-7.

21. Taghizadeh Z, Shirmohammadi M, Feizi A, Arbabi M. The effect of cognitive behavioural psycho-education on premenstrual syndrome and related symptoms.J Psychiatr Ment Health Nurs. 2013;20(8):705-13. doi: 10.1111/j.1365-2850.2012.01965.x. [PubMed: 22957993].

22. Nazari H, Hashemian K, Poornikdast S, Birashk B, Ghasemzadeh R, Alhaviradi Yani X. Effectiveness of cognitive-behavioral therapy on symptoms of premenstrual syndrome. Med J Islamic Republic Iran. 2011;30(2):144-9.

23. Salamat S, Ismail KMK, O' Brien S. Premenstrual syndrome. Obstetr Gy naecol Reprod Med. 2008;18(2):29-32. doi:10.1016/j.ogrm.2007.11.006.

24. Hadi N, ROSTAMI GN. Determinant factors of medication compliance in hypertensive patients of Shiraz, Iran. Arch Iranian Med. 2004;7(4):292-6. 